www.jmscr.igmpublication.org Impact Factor 5.244

Index Copernicus Value: 83.27 ISSN (e)-2347-176x ISSN (p) 2455-0450 crossref DOI: _https://dx.doi.org/10.18535/jmscr/v4i11.25

\title{
Congenital High Airway Obstruction - Case Report with Imaging Features
}

\author{
Authors \\ Dr Avinash Naikwadi ${ }^{1}$, Dr Rujuta N. Rege ${ }^{2}$ \\ ${ }^{1}$ National Hospital \\ ${ }^{2}$ Saveetha Medical College and Hospital \\ Email: rujuta.rege@hotmail.com
}

\begin{abstract}
Congenital high airway obstruction syndrome (CHAOS) is a near fatal condition of multifactorial inheritance, due to the obstruction of the fetal upper airways, which may be partial or complete in which the fetus has a dilated trachea, enlarged echogenic lungs, an inverted or flattened diaphragm, and ascites. Prenatal recognition of the disease is quite important due to the recently described management options We report here a case of CHAOS due to laryngeal atresia diagnosed by antenatal ultrasonography at 19 weeks of gestation. We also briefly review the relevant literature with the associated management options.
\end{abstract}

\section{Case}

A 22-year-old primigravida woman was referred for routine second trimester antenatal ultrasound at 19 weeks of gestation. There was no history of consanguinity and the family history was unremarkable.

Ultrasound examination revealed that the fetus had distinctly dilated trachea, bilateral enlarged hyperechoic lungs, inferiorly displaced and flattened diaphragms [Figure 1]. The heart was centrally placed and appeared to be compressed by the enlarged lungs and gross fetal ascites [Figure 2]. Increased nuchal thickness was also noted [Figure 3]. No other additional anomaly was found.

Based on ultrasound examination, diagnosis of CHOAS due to laryngeal atresia was made. After thorough counselling and explaining the outcomes of this pregnancy the parents were convinced to undergo termination as this was incompatible with life and was also fitting the criteria for legal age of termination.

\section{Discussion}

Laryngeal atresia is a very rare congenital malformation which results due to nondevelopment of the $6^{\text {th }}$ branchial arch during embryogenesis resulting in a clinical spectrum defined as congenital high airway obstruction syndrome (CHAOS) ${ }^{[1,2]}$. This has been classified into three types by Smith and Bain: type 1- There is complete atresia of the larynx with midline fusion of the arytenoid cartilages and intrinsic muscles; type 2 - There is infra-glottic obstruction characterized by a dome-shaped cricoid cartilage obstructing the lumen; and type 3 - in which there is occlusion of the anterior fibrous membrane and fusion of the arytenoid cartilages at the level of the vocal processes. ${ }^{[1]}$ Normally during the course of pregnancy, the fluid secreted by fetal lung is absorbed through the tracheobronchial tree. 
However, the non-clearance of fluid from the lungs results in parenchymal hyperplasia, which appears as enlarged hyperechoic lungs on ultrasonography thus resulting in gradual increase of intra-tracheal pressure leading to enlargement of the lungs. This leads to a series of sequential events that occur in the fetus namely; enlargement of the lungs which cause compression of the heart and great veins. Due to this compression, the heart becomes small, compressed and thus dysfunctional. Decreased venous return and a dysfunctional cardiovascular system lead to ascites and hydrops. The diaphragm flattens or inverts depending on the severity of the process ${ }^{[3] .}$ Besides laryngeal atresia, the other rare underlying causes of CHAOS are tracheal atresia, laryngeal agenesis, laryngeal webs or cysts and subglottic stenosis or atresia. However, the obstruction is mostly secondary to laryngeal atresia $^{[4,5]}$. Association of laryngeal atresia with partial trisomy 9 and 16, resulting in maternal translocation has also been reported. ${ }^{[6,7]}$

The main diagnostic tool for prenatal diagnosis of CHAOS is sonography which has typical findings on evaluation. As mentioned earlier bilateral large hyperechoic lungs, small, compressed, and centrally placed heart, flattened or inverted diaphragm, and ascites are characteristic findings on sonographic examination. ${ }^{[4]}$ In this case the fetus also had the presence of increased nuchal thickness of $5.3 \mathrm{~mm}$.

Regarding the amniotic fluid index, compression of the esophagus by widened airways may lead to polyhydramnios, as the fetal swallowing of the fluid is disrupted. On the other hand, impaired swallowing of the fetus may also cause oligohydramnios ${ }^{[3]}$. In our case however there were no signs of polyhydramnios as the gestational age of the fetus was only 19 weeks. Ultrasonography is first-line diagnostic imaging tool owing to its low cost and wide spread use. However, in case if any fetal surgical intervention is planned, MR imaging can be used more effectively for detecting the exact level of obstruction ${ }^{[8]}$.
Congenital high airway obstruction syndrome should be differentiated from extrinsic causes of tracheaolaryngeal obstruction. Some of these extrinsic causes are lymphatic malformation, cervical teratoma, and vascular rings like double aortic arch ${ }^{[2,4]}$. CHAOS is mostly sporadic, and the exact incidence is not known ${ }^{[2,3,9]}$.

Due to the recent advances in technology fetuses diagnosed as CHAOS have options available to be treated in-utero. If $\mathrm{CHAOS}$ due to incomplete obstruction is diagnosed in the late $2^{\text {nd }}$ or in the $3^{\text {rd }}$ trimester in the absence of hydropse, the EXIT procedure (ex-utero intrapartum treatment) can be offered. The main aim of the procedure is to secure an intact airway for the fetus before the feto-maternal circulation is stopped ${ }^{[10]}$.

\section{Conclusion}

Hence the necessity of detailed examination and complete evaluation of all suspected patients due to possibility of coexistence of any genetic syndrome and significant implications of inheritance for future pregnancies ${ }^{[11]}$.

As a result, CHAOS is a rare and fatal cause of congenital airway obstruction if unrecognized during prenatal period. Antenatal sonographic imaging is the principle modality showing typical findings which can lead to a diagnosis. MRI is superior to sonography in demonstrating the level of obstruction and in assisting in the differential diagnosis by excluding extrinsic causes of obstruction. This is important especially if any fetal intervention is considered. 


\section{JMSCR Vol||04||Issue||11||Page 13671-13674||November}

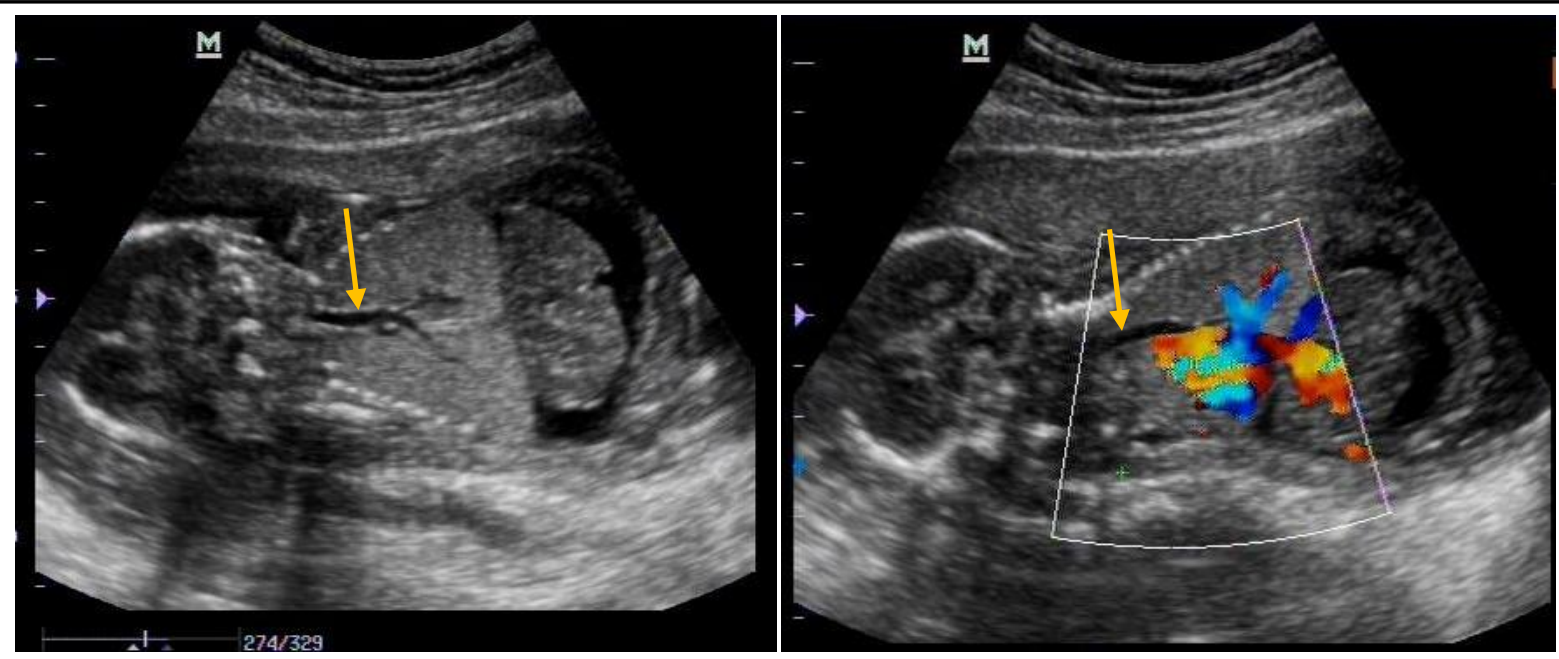

Fig 1 :- USG of the fetus in the coronal plane, at the level of the thorax shows a dilated trachea (yellow arrow), bilateral enlarged hyperechoic lungs, the flattened diaphragm with fetal ascites.

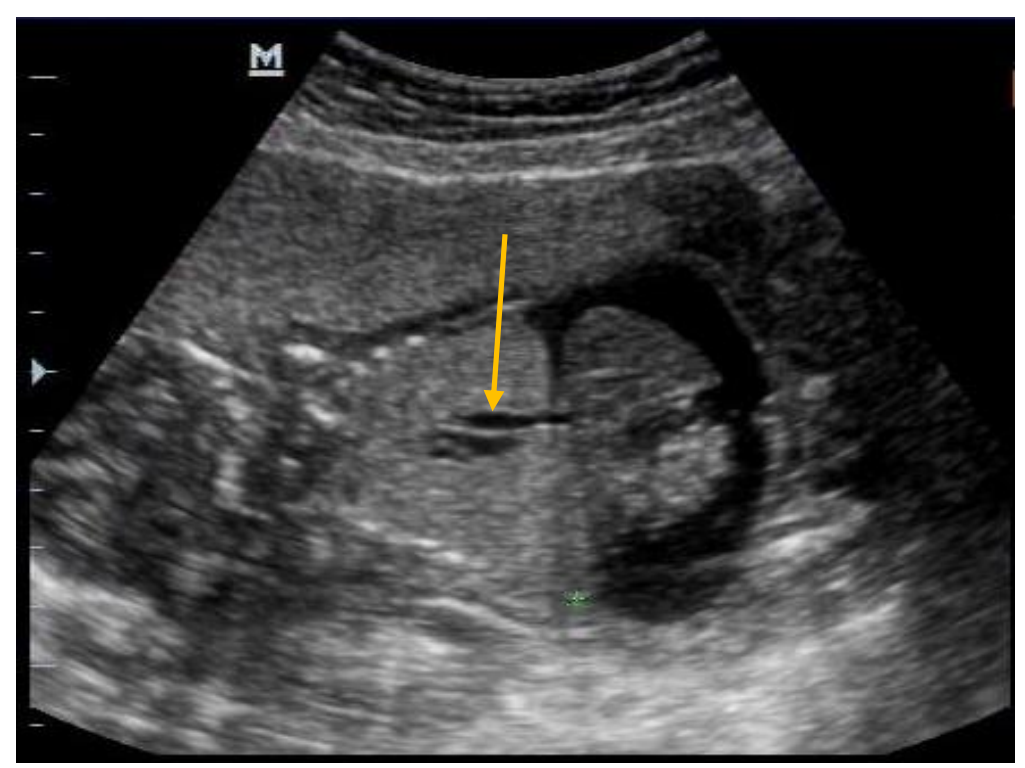

Fig 2 :- USG of the fetus in the coronal plane, at the level of the thorax shows heart compressed and centrally placed (yellow arrow ) with presence of fetal ascites.
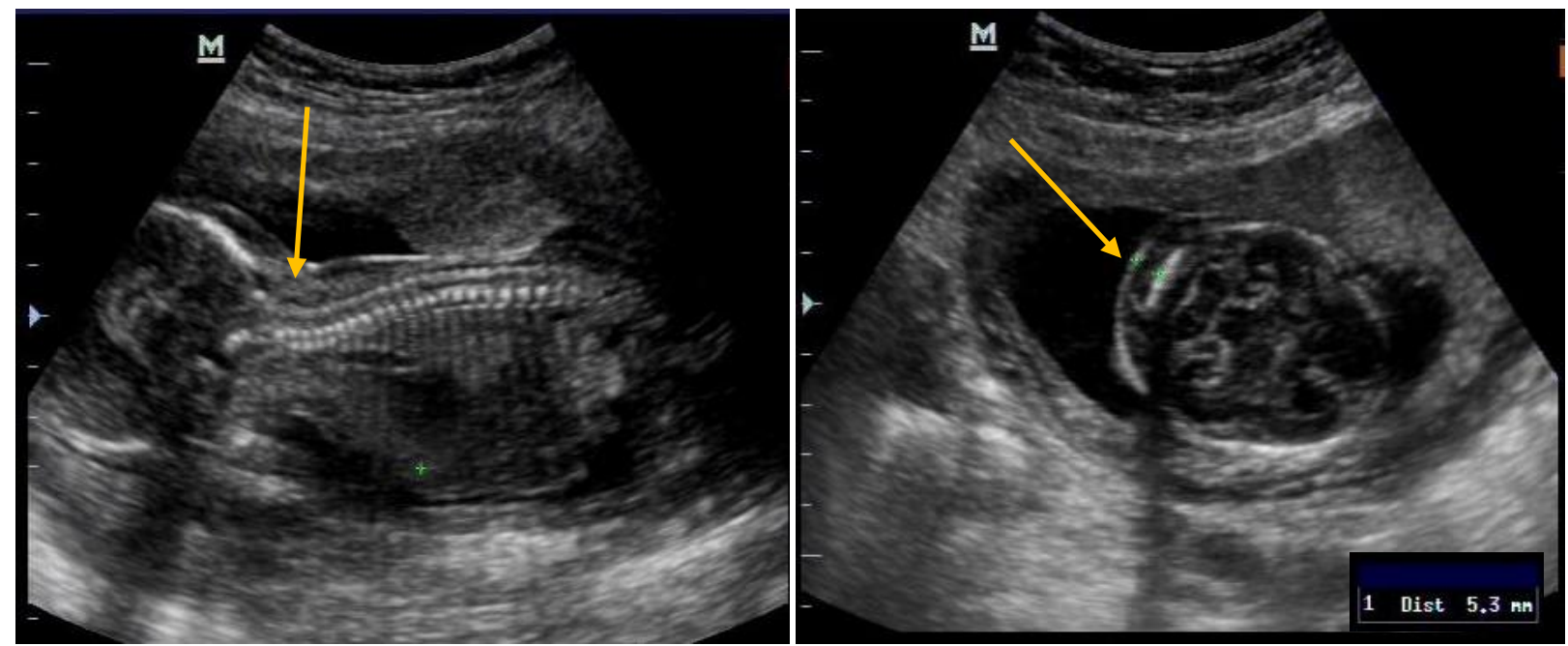

Fig 3 :- USG of the fetus in the sagittal and axial view show increased nuchal thickness (yellow arrow ) measuring upto $5.3 \mathrm{~mm}$. 


\section{References}

1. Smith II, Bain AD. Congenital atresia of the larynx: A report of nine cases.Ann Otol Rhinol Laryngol. 1965;74:33849. [PubMed]

2. J. L. Roybal, K. W. Liechty, H. L. Hedrick et al., "Predicting the severity of congenital high airway obstruction syndrome," Journal of Pediatric Surgery, vol.45,no.8,pp.1633-1639,2010.

3. P. Joshi, L. Satija, R. A. George et al., "Congenital high airway obstruction syndrome-antenatal diagnosis of ararecase of airway obstruction using multimodality imaging," Medical Journal Armed ForcesIndia,vol.68,no.1,pp.78-80,2012.

4. M. Garg, "Case report: antenatal diagnosis of congenital high airway obstruction syndrome. Laryngeal atresia,'Indian Journal of Radiologyand Imaging, vol.18,no.4,pp.350-351,2008.

5. J. Courtier, L. Poder, Z. J. Wang, A. C. Westphalen, B. M. Yeh, and F. V. Coakley, "Fetal tracheolaryngeal airway obstruction: prenatal evaluation by sonogramphy and MRI," Pediatric Radiology, vol.40,no.11,pp.1800-1805,2010.

6. Baker DC, Jr, Savetsky L. Congenital partial atresia of the larynx. Laryngoscope. 1966;76:616-20. [PubMed]

7. Van den Boogaard MJ, De Pater J, Hennekam RC. A case with laryngeal atresia and partial trisomy 9 due to maternal 9;16 translocation. Genet Couns.1991;2:83-91. [PubMed]

8. F.V.Coakley,H.Hricak,R.A.Filly,A.J.Bark ovich,andM.R. Harrison, "Complex fetal disorders: effect of MR imaging on management: preliminary clinical experience," Radiology, vol. 213,no.3,pp.691696,1999.

9. P. Vanhaesebrouck, K. De Coen, P. Defoort et al., "Evidence for autosomal dominant inheritance in prenatally diagnosed CHAOS," European Journal of
Pediatrics, vol. 165, no. 10, pp. 706708,2006.

10. P.Walker,J.Cassey,andS.O'Callaghan,“Ma nagementofantenatallydetectedfetalairway obstruction,'International Journal of Pediatric Otorhinolaryngology, vol. 69, no. 6, pp. 805-809, 2005.

11. Y.Lim,T.M.Crombleholme,H.L.Hedricket al.,"Congenital high airway obstruction syndrome: natural history and management," Journal of Pediatric Surgery, vol. 38, no. 6, pp. 940-945,2003 